\title{
Plastic Deformations of Measured Object Surface in Contact with Undeformable Surface of Measuring Tool
}

\author{
Marek Kowalik ${ }^{1}$, Mirosław Rucki ${ }^{1}$, Piotr Paszta², Rafał Gołębski ${ }^{2}$ \\ ${ }^{1}$ Kazimierz Pulaski University of Technology and Humanities, Mechanical Faculty, \\ Malczewskiego 29, 26-600 Radom, Poland, m.kowalik@uthrad.pl \\ ${ }^{2}$ Czestochowa University of Technology, Faculty of Mechanical Engineering and Computer Sciences, \\ Aleja Armii Krajowej 21,42-201, Czestochowa, Poland
}

\begin{abstract}
Measuring errors caused by deformation (flattening) of a measured object appear under the influence of pressure force and weight of the measured object. Plastic strain, arising at the contact of a measured object and an undeformable contact tip of a measuring device, can be calculated by applying the Hertz plastic solution and the hypothesis of plastic strain. In a small area of contact between two bodies pressing against one another with force $F$, there appears the so-called contact stress. It can sometime reach very high values, exceeding the yield point, even when the contact pressure is relatively small. In the present work, the authors describe a theoretical solution to the problem of plastic strain between two bodies. The derived relationships enable to calculate force $F$ during measurements of a deformable object by means of an instrument with an undeformable, spherical measuring tip. By applying the $\tau_{\max }$ hypothesis, a solution was obtained for the force $F$ in an inexplicit form. The theoretical solution was verified with the digital simulation and experimental measurement. With the FEM method, the limit length gage was modeled in interaction with the measured shaft of a diameter $d$ larger than the nominal one of $\Delta l$ value.
\end{abstract}

Keywords: Measuring deformation, contact plastic strain, contact pressure.

\section{INTRODUCTION}

The accuracy of the measurement is affected by unavoidable deflection and deformation of the contacting surfaces under the gaging force in all measurements of contact type [1]. In reality, both the gap gage and the measured detail underwent deformation [2], but in the simplified model the plastic deformations under the pressure of the undeformable stylus could be calculated with the elastic solution proposed by H. Hertz and the hypothesis of the plastic deformation, using, e.g., the FEM simulation method. Its verification could be performed either with simple inspection methods like air gaging [3] or with advanced 3D surface topography analysis devices [4].

When two contacting solid bodies are pressed to one another with force $F$, the contact pressure causing stresses in the material appears in the small area of the mutual contact. Even though the gaging force is low, the small areas of the contact points develop high contact pressures [5]. The local force sometimes rises up substantially and even crosses the plastic deformation limits despite the fact that the force itself is relatively small.

This problem appears especially in case of all categories of fixed gages of high stiffness. The contact of the fixed gage with the measured object takes place on the small surface, which causes high gaging pressure. The construction of the fixed gage and the geometrical profile of the measured object require relatively small force applied to the fixed gage by the operator. However, it may result in large contact pressure on the small surface, which leads to permanent plastic deformation of the measured surface [6]. The same problem appears also in the automation of the manufacturing processes. Contact conditions are critical to achieve the adequate force of measuring robot grippers [7], [8]. Too high contact pressure and inappropriate shape of the grip end may cause undesirable flattening of the surface structure of the workpiece, especially in case of soft metals.

In the present work, a limit length gage was taken as an example of such a measurement in order to assess the plastic deformation of the shaft surface within a value equal to the roughness height. First, the shaft was prepared, so that its diameter was close to the upper tolerance. Then it was measured with the fixed gage, so that some force had to be applied. Next, in order to assess the deformation caused by the contact pressure, a profilogram was made.

Because the deformations caused by the external factors and weight of the measured objects are very small, the respective surface is very small, too. It was assumed for the calculation and MES simulation purposes that both surfaces 
and the body of the limit length gage are undeformable. In order to obtain clearly distinguishable results, the material of the measured shaft was chosen of lower strength. Harder materials of higher $R_{e}$ are suffering plastic deformation from the measurement force in smaller degree. On the other hand, harder material would cause substantial impact on the fixed gage, which would no more be considered undeformable. In the calculation example, the force was determined which would cause the plastic deformation of the measured shaft of a value $0.01 \mathrm{~mm}$.

\section{THE CONTACT PRESSURE CALCULATION}

According to the theory proposed by $\mathrm{H}$. Hertz, in two objects of regular profile curvature, the component stress along $z$-axis, for $x=y=0$ (Fig.1.) becomes the main one and can be calculated from the following formulae (1) [9].

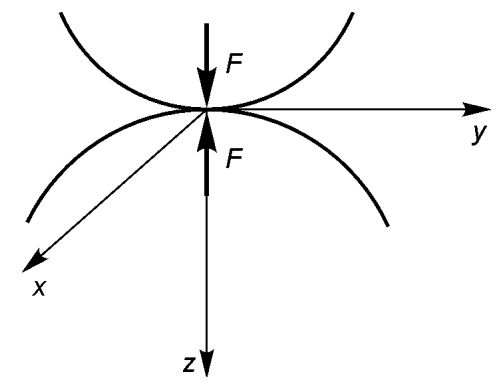

Fig.1. Scheme of contact of two bodies with regular shapes, the squeezing force $F$, according to $\mathrm{H}$. Hertz (general case).

$$
\begin{gathered}
\sigma_{r}=\sigma_{1}=\frac{1}{2} q\left[\frac{2(1+v) \cdot z}{\sqrt{a^{2}+z^{2}}}-(1+2 v)-\left(\frac{z}{\sqrt{a^{2}+z^{2}}}\right)^{3}\right] \\
\sigma_{z}=\sigma_{2}=q\left[\left(\frac{z}{\sqrt{a^{2}+z^{2}}}\right)^{3}-1\right],
\end{gathered}
$$

where $\sigma_{r}, \sigma_{z}$ - the radial and axial stresses corresponding with the main stresses $\sigma_{1}, \sigma_{2} ; v$ - Poisson's number, $a-$ contact substitute radius according to Hertz, $q$ - pressure according to Hertz.

In the contact area and the adjacent ones, there appears the 3D tension state. Thus, the pressure force calculations could be based on the respective strain hypotheses. The material of the measured object can receive the stresses of the values above the yield strength, which defines the plastic limit. It may be explained by the fact that in the pressured points, there is the strain state calculated for the three-axes squeeze. Hence, if the maximal stress $\tau_{\max }$ hypothesis (CoulombTresca-Guest) [10], [11] is applied, the contact pressure $F$ could be calculated as follows:

$$
\tau_{\max }=\frac{\sigma_{1}-\sigma_{2}}{2} .
$$

After putting (1) to (2), it is received:

$$
\frac{\sigma_{1}-\sigma_{2}}{2}=\frac{1}{2} q\left[\frac{2(1+v) \cdot z}{\sqrt{a^{2}+z^{2}}}-(1+2 v)-\left(\frac{z}{\sqrt{a^{2}+z^{2}}}\right)^{3}-2\left(\frac{z}{\sqrt{a^{2}+z^{2}}}\right)^{3}+2\right]
$$

After proper transformations, the values $\tau_{\max }=0.5 R_{e}$, $z=2 R z_{\text {max }}$ and $q=q_{0}=\frac{3 F}{2 \pi a^{2}}$, could be included into (3), where $R_{e}$ - yield stress, $R z_{\max }$ - maximal asperity height. Then the equation will take the following form:

$$
0.5 R_{a}=\frac{3 F}{2 \pi a^{2}}\left[-\frac{1+2 v}{2}+(1+v) \frac{2 R z_{\max }}{\sqrt{a^{2}+4 R z_{\text {ax }}^{2}}}-\frac{3}{2}\left(\frac{2 R z_{\max }}{\sqrt{a^{2}+4 R z_{\operatorname{ma}}^{2}}}\right)^{3}+1\right]
$$

From here, the equation for the force $F$ could be written as (4) below:

$$
F=\frac{\pi a^{2} R_{e}}{3}\left[-\frac{1+2 v}{2}+(1+v) \frac{2 R z_{\max }}{\sqrt{a^{2}+4 R z_{\max }^{2}}}-\frac{3}{2}\left(\frac{2 R z_{\max }}{\sqrt{a^{2}+4 R z_{\max }^{2}}}\right)^{3}+1\right]^{-1} .
$$

In the equation (4), $a$ becomes the substitute semi-axis of the ellipse and the $b$ semi-axis appears: $a=\sqrt{a^{\prime} \cdot b^{\prime}}$. It may be calculated as follows:

$$
a=\sqrt[3]{\frac{3\left(1-v^{2}\right) F}{2 E \cdot \sum_{(i)} k_{i}}}
$$

where $E$ - Young's modulus.

On the other hand, $\sum_{i} k_{i}=\sum_{1}^{2} k_{p}+\sum_{1}^{2} k_{k}$, where $k_{p}-$ curvature of the measured object profile, $k_{k}$ - curvature of the measurement stylus, so $\sum_{(i)} k_{i}$ - the values of the curvatures of the typical measurements described in detail,

e.g., in [12]. The equation of implicit form is obtained, because force $F$ is determined by two equations: (4) and (5).

The force may be calculated from them using the digital simulation, and for that purpose it was introduced to the numerical solution as the replacement of $a=2 x R z_{\text {max }}$, then

$$
\frac{2 R z_{\max }}{\sqrt{a^{2}+4 R z_{\max }^{2}}}=\frac{1}{\sqrt{1+x^{2}}} \text {. }
$$

After it is summed up

$1-\frac{1+2 v}{2}=\frac{-1-2 v+2}{2}=\frac{1-2 v}{2}$, then (4) for $F$ will take the form:

$$
F=\frac{\pi a^{2} R_{e}}{3}\left[\frac{1-2 v}{2}+(1+v) \frac{1}{\sqrt{1+x^{2}}}-\frac{3}{2}\left(\frac{1}{\sqrt{1+x^{2}}}\right)^{3}\right]^{-1}
$$


Equation (5) may be transformed into the below form:

$$
a=\sqrt[3]{\frac{3(1-v)^{2} F}{2 E \cdot \sum_{(i)} k_{i}}}=\sqrt[3]{F} \cdot \sqrt[3]{\frac{3(1-v)^{2}}{2 E \cdot \sum_{(i)} k_{i}}}=\sqrt[3]{F} \cdot A,
$$

where $A$ may be assumed for the analyzed process as a known value, because $v, E$ and $\sum_{(i)} k_{i}$ are unequivocally determined. When two formulae (6) and (7) are compared, it could be derived:

$$
\begin{gathered}
F=\left(\frac{a}{A}\right)^{3}=\frac{\pi a^{2} R_{e}}{3}\left[\frac{1-2 v}{2}+(1+v) \frac{1}{\sqrt{1+x^{2}}}-\frac{3}{2}\left(\frac{1}{\sqrt{1+x^{2}}}\right)^{3}\right]^{-1} \\
\frac{1}{A^{3}}=\frac{\pi R_{e}}{3 a}\left[\frac{1-2 v}{2}+(1+v) \frac{1}{\sqrt{1+x^{2}}}-\frac{3}{2}\left(\frac{1}{\sqrt{1+x^{2}}}\right)^{3}\right]^{-1} \\
\frac{1-2 v}{2}+(1+v) \frac{1}{\sqrt{1+x^{2}}}-\frac{3}{2}\left(\frac{1}{\sqrt{1+x^{2}}}\right)^{3}=\frac{\pi R_{e} A^{3}}{3 a} .
\end{gathered}
$$

After summation and transformation of some parts, which is shown below, (8) could be rewritten [9]:

$$
\begin{gathered}
\frac{(1+v)}{\sqrt{1+x^{2}}}-\frac{3}{2}\left(\frac{1}{\sqrt{1+x^{2}}}\right)^{3}=\frac{2(1+v)\left(1+x^{2}\right)-3}{2\left(\sqrt{1+x^{2}}\right)^{3}}=\frac{v-\frac{1}{2}+(1+v) x^{2}}{\left(1+x^{2}\right)^{\frac{3}{2}}} \\
-\frac{1-2 v}{2}=v-\frac{1}{2} \\
\frac{\pi R_{e} A^{3}}{3 a}+\left(v-\frac{1}{2}\right)=\frac{(1+v) x^{2}+v-\frac{1}{2}}{\left(1+x^{2}\right)^{\frac{3}{2}}}
\end{gathered}
$$

However, when $a=2 x R z_{\max }$, then

$$
\frac{\pi R_{e} A^{3}}{6 x R z_{\max }}+\left(v-\frac{1}{2}\right)=\frac{(1+v) x^{2}+v-\frac{1}{2}}{\left(1+x^{2}\right)^{\frac{3}{2}}}
$$

Multiplying by $x$, the result is

$$
\frac{\pi R_{e} A^{3}}{6 R z_{\max }}+\left(v-\frac{1}{2}\right) x=\frac{(1+v) x^{2}+v-\frac{1}{2}}{\left(1+x^{2}\right)^{\frac{3}{2}}} x
$$

Assuming $B=\frac{\pi R_{e} A^{3}}{R z_{\max }}$ and squaring both sides of the equation, (8b) becomes the polynomial of $8^{\text {th }}$ degree.
Because the parameter $B$ is determined unequivocally with known values of $R_{e}, v, E$ and $\sum_{(i)} k_{i}$ as well as the given roughness value $R z_{\max }$, the value $x$ can be calculated:

$$
\left[B+\left(v-\frac{1}{2}\right) x\right]^{2}\left(1+x^{2}\right)^{3}=\left[(1+v) x^{2}+v-\frac{1}{2}\right]^{2} x^{2}
$$

With the available computer program (e.g., Mathematica, Derive, etc.), the variable $x$ could be calculated from (9), choosing the correct square root from the solutions. When the appropriate $x$ is ascribed to the given roughness $R z_{\max }$, the pressure force can be calculated, which causes the plastic deformation in the roughness dimensions during the measurement:

$$
F=\left(\frac{a}{A}\right)^{3}=\left(\frac{R z_{\max } \cdot 2 x}{A}\right)^{3}
$$

This way, the pressure force $F$ value was found, which caused the permanent deformation of the measured object in the contact area with the undeformable surface of the fixed gage. The parameter $A$, which appears in (10) is dependent on the Poisson's number, Young's modulus and the values of the contacting surface curvatures. The material properties described by (6) and (7) are rather implicit ones, but the parameter $A$ could be derived as a simple function.

\section{Calculation EXAmple}

Fig.2. presents the scheme of two objects' contact during the measurement of the shaft diameter $d$ with the limit length gage. The pressure force $F$ should be calculated, and the force $Q$ applied to the gage. The values were: $d=50$ $\mathrm{mm} ; R z_{\max }=0.01 \mathrm{~mm} ; \quad R_{e}=300 \mathrm{MPa} ; \quad E=2.1 \cdot 10^{5} \mathrm{MPa}$; $v=0.3$.

The pressure force $F$ is calculated form the formula (10) with previous calculation of $x$ from the polynomial (9). Then $A$ and $B$ are calculated from the below equations:

$$
B=\frac{\pi R_{e} A^{3}}{6 R z_{\max }}, \text { where } A=\sqrt[3]{\frac{3(1-v)^{2}}{2 E \cdot \sum_{(i)} k_{i}}}
$$

After the values are introduced, it is calculated as follows:

$$
B=\frac{\pi R_{e}}{6 R_{2}} \cdot \frac{3(1-v)^{2}}{2 E \cdot \sum_{(i)} k_{i}}=\frac{\pi \cdot 300}{8 \cdot 0.01} \cdot \frac{0.7^{2} \cdot 50}{2.1 \cdot 10^{5}}=\frac{\pi \cdot 2.45 \cdot 3 \cdot 10^{5}}{1.68 \cdot 10^{5}}=\frac{23.8}{16.8}=1.37375 \approx 1.4 .
$$

$$
A^{3}=\frac{3}{2} \cdot \frac{(1-v)^{2}}{E \cdot \sum_{(i)} k_{i}}=\frac{3}{2} \cdot \frac{0.7^{2} 50}{2.1 \cdot 10^{5}}=1.75 \cdot 10^{-4} .
$$


Then the polynomial takes the following form:

$$
\begin{gathered}
(1.4+0.2 x)^{2}\left(1+x^{2}\right)^{3}=\left(1.3 x^{2}+0.2\right)^{2} \cdot x^{2}, \\
(7+x)^{2}\left(1+x^{2}\right)^{3}-\left(7.5 x^{2}+1\right)^{2} \cdot x^{2}=0 .
\end{gathered}
$$

Then, from the computer solution the approximated positive square roots are obtained $x \in\{0,9,4,9,6,2,7\}$. Putting the largest value $x=9$ to the $F$ formula, it is obtained:

$$
F=\left(\frac{x \cdot 2 R z_{\text {max }}}{A}\right)^{3}=\frac{(9 \cdot 2 \cdot 0.01)^{3}}{1.75 \cdot 10^{-4}}=33.3 \mathrm{~N} .
$$

From Fig.2., the relation between the forces $Q$ and $F$ is as follows:

$$
Q=2 F \operatorname{tg}(\alpha+\rho)=33.3 \cdot 2 \operatorname{tg}\left(11^{\prime \prime} 19^{\prime}+1^{\prime} 36^{\prime}\right) \approx 16 \mathrm{~N},
$$

where $\alpha$-the sliding angle and $\rho$ - the friction angle, both dependent on the shaft dimensions and its deformation during the measurement.

It should be noted that the obtained result confirms quite reasonably the theoretic solution of the formula (10) giving the $F$ force as dependent on the deformation of the measured object.

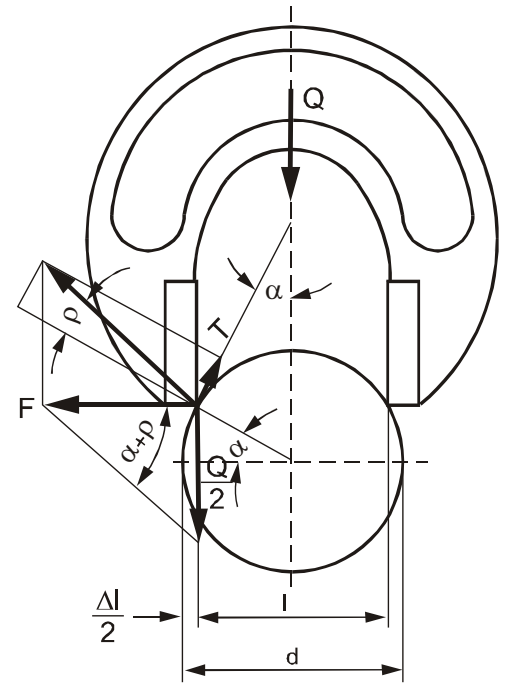

Fig.2. Limit length gage in contact with a shaft of diameter larger than $d$ with $\Delta l: Q$ - the operator's force; $T$ - the friction force; $F$ the force normal to the surface of the gage surface; $\rho$ - the friction angle; $\alpha$ - the sliding angle.

The simulations were performed in the system Ansys Workbench 14.0, using the module "static structural." The geometry model was built with Solid Edge ST5, considering the actual diameter of the shaft $d=50 \mathrm{~mm}$. The deformation condition was assumed to be 3-dimensional. The grid was made out of 4-sided elements for overall dimensions of the grid $0.5 \mathrm{~mm}$ [13]. Then the grid density was increased in the contact area using the tool "sphere of influence" with the cell dimensions of $0.01 \mathrm{~mm}$. Then the conditions of the contact were set as follows: the contact surfaces and the body of the gage were assumed as undeformable (Rigid body - Ansys), friction between the elements was assumed negligibly small (the appropriate tool was used which was "friction less support" - Ansys). The standard elastic and plastic model of the matter was taken like in the papers [14], [15] from the Ansys library for "structural steel." In the simulation, we assumed an elasto-plastic model of material with nonlinear work hardening described by the LudwikHollomon exponential equation $\sigma=C \varepsilon^{n}$, where $C$ - workhardening coefficient, $n$ - work-hardening exponent. This relationship is commonly used in simulation programs of the processes of cold plastic working, and it proves sufficiently accurate. For the simulation, the exponent $n=0.15$ was chosen. The other data were assumed the same as in theoretical calculations:

$$
R_{e}=300 \mathrm{MPa} ; E=2.1 \cdot 10^{5} \mathrm{MPa} ; v=0.3 .
$$

In Fig.3. the results of measured surface deformation with undeformable limit length gage simulation are presented. In the contact area, the stresses cross the plasticity limits and cause the plastic deformation. Fig.4. presents the results of the overall deformation simulations in the contact area between the gage and the shaft surface.

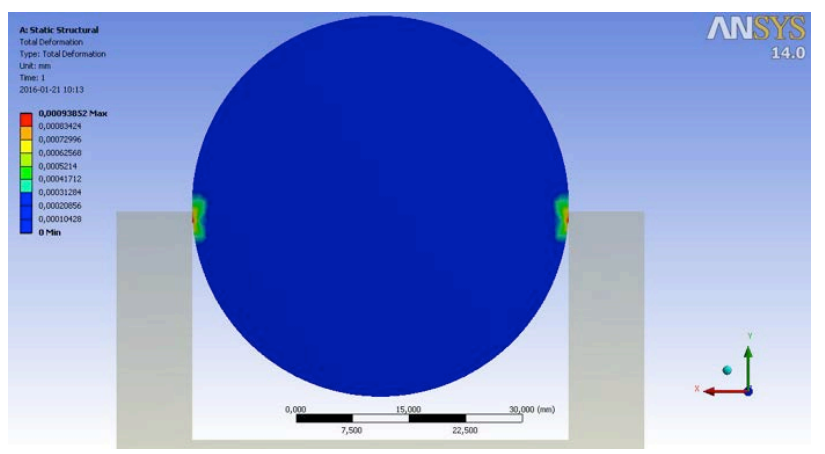

Fig.3. Diagram of the material deflection during the measurement of the shaft $d=50 \mathrm{~mm}$ with the limit length gage, calculated against criteria of maximal reduced strain proposed by Huber - Mises - Hencky.

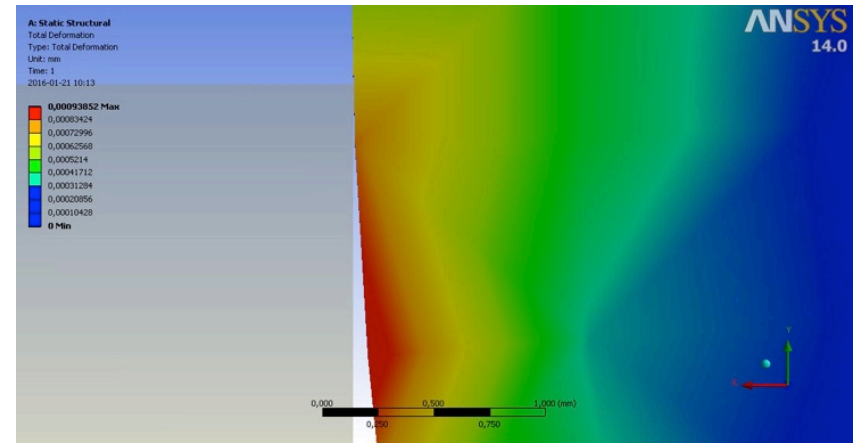

Fig.4. Diagram of the material deflection in the contact area of the gage surface with the shaft, calculated against criteria of maximal reduced strain proposed by Huber - Mises - Hencky. 
For the same data concerning the material, the simulation analysis was performed for the shaft diameters $10 \div 50 \mathrm{~mm}$ forcing them into the undeformable bounds that were $0.01 \mathrm{~mm}$ smaller than the diameter of the examined shaft. Such conditions correspond with the reality when the measured shaft slightly crosses the upper tolerances. In all simulated cases, some plastic deformation took place in the contact area. Fig.5. presents the graph with the deformation values. It is seen that the deformation is almost the same for any simulated diameter. It could be explained considering that the deformation took place in a very small surface area, so the shaft diameter has negligible impact on its value.

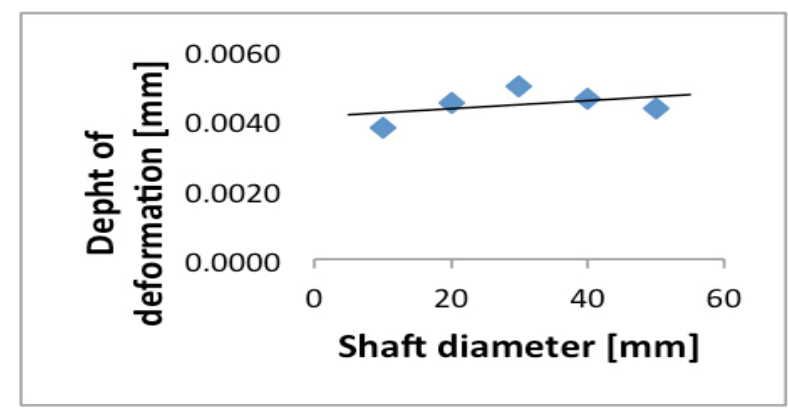

Fig.5. FEM simulation results for the deformed shafts of diameters $10 \div 50 \mathrm{~mm}$.

There is no need to analyze the trend revealed in Fig.5. between 0.004 and $0.005 \mathrm{~mm}$ of the deformation depth. For the measuring task performed by the fixed gage, meant to check the dimension within the assumed tolerances, the resolution below $1 \mu \mathrm{m}$ is not required.

\section{EXPERIMENTAL VERIFICATION}

To correlate the simulation results with the real deformation caused by the limit length gage, the following experiment was performed. The series of shafts were made out of steel with plasticity $R_{e}=300 \mathrm{MPa}$, like in the case of FEM simulations, roughness $R a=3.47 \mu \mathrm{m}$ and $R a=6.47 \mu \mathrm{m}$.

The diameter of the shaft was made $50.010 \pm 0.002 \mathrm{~mm}$ compared to the $50 \pm 0.001 \mathrm{~mm}$ dimension of the limit length gage. Next, the shaft was manually put into the gage. In the contact area, there appeared the reflecting imprint indicating plastic deformation of the surface layer. In Fig.6. there is a photograph, where the contact zone is visibly different from the rest of the shaft surface. The plastic deformation caused by measurement looks distinguishably brighter. The depth of the contact zone (ca. $5 \mu \mathrm{m}$ ) related to the rest of the undeformed shaft surface is clearly seen in the profilograms in Fig.7.

It was assumed that neither in-depth analysis of the 3D topography characteristics was required, nor its impact on the roughness parameters [16]. To assess the depth of deformation, the measurement was made with the profilometer. The vertical zoom was set $\times 1000$ because the deformation of several micrometers was expected. The horizontal zoom was set $\times 5$ in order to distinguish the width of the limit length gage $(9 \mathrm{~mm})$. The examples of the obtained profilograms are presented in Fig.7. The mean distances between asperities are the result of the machining technology, especially feed and the cutting edge geometry. Form the profiles it is seen that these values are ca. $0.3 \mathrm{~mm}$ (Fig.7.a) and $0.1 \mathrm{~mm}$ (Fig.7.b).

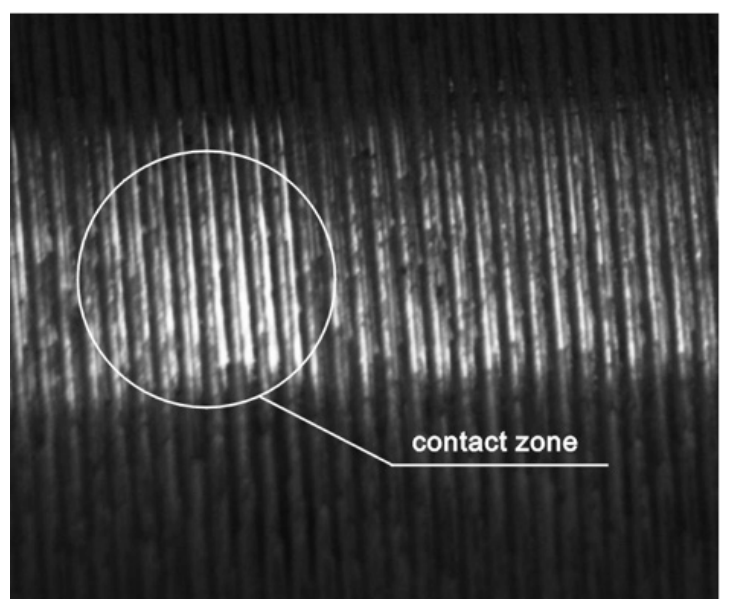

Fig.6. The photograph of the surface after the experiment $($ zoom $\times 7)$.



Fig.7. Profilograms of the deformed surface: a) the area of the formed peaks of the surface $R a=6.47 \mu \mathrm{m}$; b) the area of the surface $R a=3.47 \mu \mathrm{m}$.

In Fig.7. the lines corresponding with the peaks of the roughness and the ones corresponding with the peaks in the deformed area were added. It is clearly seen that the measuring force caused the distinguishable and measurable deformation in the $9 \mathrm{~mm}$ long contact zone. In case of roughness, both $R a=3.47 \mu \mathrm{m}$ and $R a=6.47 \mu \mathrm{m}$, the depth of deformation was ca. $5 \div 10 \mu \mathrm{m}$. Those deformations were the effect of the typical inspection made by the operator, with no additional force applied or even specific resistance noted by the operator.

The experimental results were very similar to the ones obtained by the FEM simulation. They confirmed that the deformation depth does not depend on the diameter of the measured shaft, and it takes place only in the surface area of asperities. 


\section{CONCLUSIONS}

In case of the contact measurement, the stylus or the fixed gage surfaces are in contact with the measured surface in a very small area. Thus, very large values of contact pressure appear, especially in case of the fixed gages measurement, when dimensions of the measured detail are close to the upper tolerance or cross it to a very small extent. Of great importance is also the geometric profile of the measured detail and the construction details of the applied fixed gage, which may cause very high contact pressure on the measured surface even though the operator does not apply extensive force to the gage.

The theoretical analysis performed for the limit length gage measurement of the shaft diameter $d=50 \mathrm{~mm}$ found that there may appear permanent plastic deformation of 0.01 $\mathrm{mm}$. The MES simulation confirmed that such deformation is, indeed, possible. However, the deformation values obtained from the simulation were substantially lower than those from theoretical calculation according to the theory proposed by Hertz. The possible difference may lay in the fact that the theoretical calculations do not include the phenomenon of material strengthening. In turn, the experimental results are very close to the FEM simulation. The plastic deformations of the measured shaft caused by the measuring force are located in the outer surface area. When the details are made out of stronger and harder materials, the deformations caused by the measurement or the weight of the measured element are very small. Thus, the plastic deformations in those cases are seen as scratches or rubs of the surface.

\section{REFERENCES}

[1] Curtis, M., Farago, F. (2014). Handbook of Dimensional Measurement. Industrial Press, Inc.

[2] Bialas, S., Humienny, Z., Zach, P. (2004). Verification errors due to elasticity of gap gauges. In 8th International Symposium on Measurement and Quality Control in Production: Symposium Erlangen, October 12-15, 2004. Düsseldorf: VDI Verlag, VDI-Bericht 1860, 457-469.

[3] Jermak, Cz.J., Rucki, M. (2012). Air Gauging: Static and Dynamic Characteristics. International Frequency Sensor Association (IFSA) Publishing.

[4] Wieczorowski, M., Mamalis, A.G., Rucki, M., Lavrynenko, S.N. (2008). Interferometry and scanning microscopy in asperity measurement of biomedical surfaces. Nanotechnology Perceptions, 4, 265-288.
[5] Dotson, C.L. (2016). Fundamentals of Dimensional Metrology. Cengage Learning.

[6] Messerschmidt, U. (2010). Dislocation Dynamics During Plastic Deformation. Springer.

[7] Kluz, R., Trzepiecinski, T. (2015). Analysis of the optimal orientation of robot gripper for an improved capability assembly process. Robotics and Autonomous Systems, 74, 253-266.

[8] Kluz, R., Trzepiecinski, T. (2014). The repeatability positioning analysis of the industrial robot arm. Assembly Automation, 34 (3), 285-295.

[9] Kowalik, M. (2008). Calculation of squeezing forces during longitudinal rolling for the final passage. The Archive of Mechanical Engineering, LV (2), 81-91.

[10] Bućko, S., Jodłowski, H., Trzebicki, M. (2012). Numerical-experimental analysis of plastic flow beginning phase round the hole in the thin shield under tension. Acta Mechanica et Automatica, 6 (1), 21-25.

[11] Altenbach, H, Öchsner, A. (2014). Plasticity of Pressure-Sensitive Materials. Springer.

[12] Radzevich, S.P. (2014). Generation of Surfaces: Kinematic Geometry of Surface Machining. CRC Press.

[13] Kukielka, L. (2003). Numerical Modeling: The Contact Problem of Movable Elasto/visco-plastic Body. WIT Press, 93-104.

[14] Kukielka, L. (2001). Mathematical modeling and numerical simulation of non-linear deformation of the asperity in the burnishing cold rolling operation. In Computational Methods in Contact Mechanics V. WIT Press, 317-326.

[15] Kukielka, L., Kukielka, K. (2007). Numerical analysis of the physical phenomena in the rolling process of the round thread. In Computer Methods and Experimental Measurements for Surface Effects and Contact Mechanics VIII. WIT Press, 125-134.

[16] Majchrowski, R., Marciniak, L. (2012). Difference of profile parameters due to errors of points collections simulation analysis. In $X X$ IMEKO World Congress Metrology for Green Growth, Busan, Republic of Korea, 1992-1995.

Received July 08, 2016. Accepted October 06, 2016. 